\title{
Erratum to: The current status of cancer survivorship care and a consideration of appropriate care model in Korea
}

Hye Mi Ko, Je-Ryong Kim, Jin Sun Lee

Department of Surgery and Research Institute for Medicinal Sciences, Chungnam National University College of Medicine, Daejeon, Korea

Korean J Clin Oncol 2020;16:110-118

https://doi.org/10.14216/kjco.20017

We found an error in this article. The corrected title is as follows.

Treatment options and survival outcomes in breast cancer patients with brain metastases in HER2-enriched subtype

We sincerely apologize for the inconvenience that this may have caused. 